\title{
LETTER
}

\section{Harmful misinformation}

Sir, you rightly identify the harm that is being caused by the spread of misinformation in your excellent article. ${ }^{1}$ The dental world is familiar with the work of conspiracy theorists through the activities of anti-fluoridation activists and historical dental amalgam-related scare stories.

The British Fluoridation Society is working with colleagues world-wide to develop and implement strategies to counter anti-fluoridation propaganda. We are gathering examples and analysing 'fluorophobic' sentiments in the public arena (press, conventional electronic media, on the public platform and increasingly on social media); making sure the evidence underpinning water fluoridation is up-todate and available to interested parties; and enabling supportive colleagues to make the fluoridation case effectively and working with public health and academic colleagues. We want to understand the drivers behind the beliefs and behaviours of fluoridation opponents so we can shift the paradigm in the public debate.
The BFS would welcome input from colleagues who want to be involved in this work; there's a lot to do and it is a longterm commitment if we are to take CWF forwards.

\section{Dr Ray Lowry, Hon Secretary and Treasurer British Fluoridation Society, via email}

\section{Reference}

1. Westgarth D. How dangerous is the spread of online misinformation?. BDJ In Pract 2019; 32: 10-15.

\section{FGDP(UK) and FDS publish antimicrobial prescribing guidelines for all dentists}

The Faculty of General Dental Practice UK (FGDP) and the Faculty of Dental Surgery of the Royal College of Surgeons of England (FDS) have published the third edition of Antimicrobial Prescribing in Dentistry Good Practice Guidelines.

Developed by an intercollegiate working group led by Dr Nick Palmer FDSRCS(Eng) FFGDP(UK), it updates FGDP's wellknown Antimicrobial Prescribing for General Dental Practitioners guidance to reflect changes in the evidence base since the previous edition, and covers a much wider range of conditions.

Its scope has been extended to include management of oral and dental infections by all prescribers, not only general dental practitioners but those working in secondary dental care (including trainees), specialists (including oral and maxillofacial surgeons), and those involved in dental education and research. Its recommendations are also now appropriate for all dental patients, including adults, children, the elderly and those with special needs treated in the primary and secondary care setting.

Inappropriate prescribing of antimicrobials can exacerbate the problem of antimicrobial resistance, which leads to antibiotics no longer being effective in treating even simple infections. There are serious consequences for everyone, but particularly those undergoing major surgery, chemotherapy, organ or stem cell transplants. Every year, 25,000 people across Europe, and 700,000 worldwide, die from antibiotic-resistant infections, and the UK government predicts the annual global toll could be 10 million by 2050 .

Prudent prescribing of antimicrobials can slow down the further development of antimicrobial resistance, and all healthcare prescribers play a vital role. Dentists prescribe an estimated $10 \%$ of all oral antimicrobials prescribed in England, and are required by legislation to ensure appropriate use of antimicrobials.

Published following consultation with a range of specialist societies and national dental organisations, Antimicrobial Prescribing in Dentistry - Good Practice Guidelines offers clear guidance on when to prescribe antimicrobials, what to prescribe, for how long and at what dosage.

Dr Nick Palmer, Editor of Antimicrobial Prescribing in Dentistry - Good Practice Guidelines, said: 'Antimicrobials can be an important adjunctive therapy in treating oral infection, but side effects, potential adverse reactions and the growing threat of antimicrobial resistance mean they should be prescribed judiciously. On behalf of the guideline development group, I hope dentists in all settings will find Antimicrobial Prescribing in Dentistry a useful aid to appropriate prescribing in their everyday practice.'

Ian Mills, Dean of FGDP(UK), commented: 'For 20 years, FGDP's guidance on the prescribing of antimicrobials has helped GDPs raise standards of care, and the new edition will undoubtedly continue to

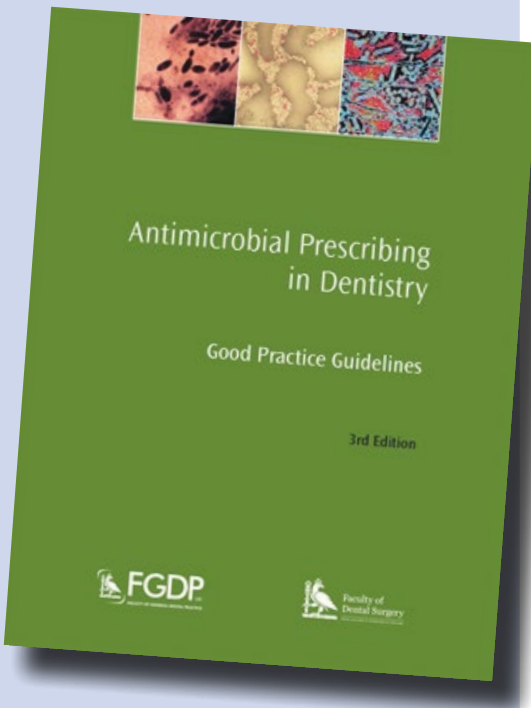

be a key reference document

for the dental team. I'd like to thank Dr Nick Palmer and his colleagues for their tireless dedication in producing it, and our colleagues at the FDS for their collaboration in extending the publication to deliver guidance for the dental profession as a whole.

Matthew Garrett, Dean of the FDS, added: 'The FDS has worked assiduously in recent years to highlight the importance of antimicrobial stewardship, and Antimicrobial Prescribing in Dentistry is an important resource to support all dentists to prescribe responsibly. The new guidance is a great example of the breadth and depth of expertise that we can bring to bear for the benefit of dental patients, and I thank all those involved in its development.' 